Meta

Journal des traducteurs

Translators' Journal

\title{
Les intermédiaires obligés du colonialisme
}

\section{Colette Touitou-Benitah}

Volume 40, numéro 1, mars 1995

URI : https://id.erudit.org/iderudit/004497ar

DOI : https://doi.org/10.7202/004497ar

Aller au sommaire du numéro

Éditeur(s)

Les Presses de l'Université de Montréal

ISSN

0026-0452 (imprimé)

1492-1421 (numérique)

Découvrir la revue

Citer cet article

Touitou-Benitah, C. (1995). Les intermédiaires obligés du colonialisme. Meta, 40(1), 15-23. https://doi.org/10.7202/004497ar d'utilisation que vous pouvez consulter en ligne.

https://apropos.erudit.org/fr/usagers/politique-dutilisation/ 


\section{LES INTERMÉDIAIRES OBLIGÉS DU COLONIALISME}

COLETTE TOUITOU-Benitah

Universild Bar-llan, Ramar-Gan, Isräl'

La colonisation française en Afrique du Nord a dû dans un premier stade faire appel aux services d'interprètes et de traducteurs. L'objectif de cet article est de délimiter cette période et de voir comment elle s'insère dans le déroulement du processus colonial puis, de faire le portrait de ces interprètes et traducteurs, de définir leurs champs d'action et leurs rapports avec le pouvoir.

\section{LA PERIODE D'ACTIVITE DES TRADUCTEURS}

Paul Cambon, Résident général de la République française en Tunisie, écrivait en 1893 dans sa préface à un recueil de lois :

Les fonctions des interprètes-traducteurs auront, surtout au début, une importance considérable $[. .$.$] Ils sont les intermédiaires obligés entre les propriétaires dont la masse est encore$ peu familiarisée avec la langue française et le tribunal mixte chargé d'appliquer la loi immobilière'.

"Surtout au début», précise Paul Cambon. Ce début de la colonisation, envisagé dans ses rapports avec la traduction. s'organise en une période pré-coloniale, une période de conquête militaire puis une période de mise en place de structures administratives et autres assurant le pouvoir à la puissance colonisatrice.

\section{La période pré-coloniale}

En amont de la colonisation, pendant des siècles et plus intensément au cours des XVII el XVIIIe siècles, l'Afrique du Nord a été l'objet de convoitise des puissances européennes. Les relations politiques se sont toujours appuyées sur des relations commerciales. Abraham I. Laredo précise que l'origine d'un des noms patronymiques des juifs du Maroc parmi les plus répandus: Tordjman, qui n'est autre que le nom arabe de la fonction d'interprète, est à chercher dans le fait que :

...au Moyen Âge et même assez récemment, il existait dans les Douanes des ports marocains des interprètes chargés des relations entre les Administrateurs du Makhzen et les Capitaines des navires et commerçants étrangers. Le titulaire de cette fonction, qui en général était remplie par un israélite. s'appelait ATURJMAN2.

Hirshberg. à propos de la période des transactions entre la maison d'Orange et le Maghreb, décrit ainsi une opération de double traduction de l'arabe en hollandais:

During that period (1680-1090) Jacob Sasportas translated diplomatic notes from Arabic into Spanish. which David Torres translated from Spanish into Dutch?.

Les riches marchands juifs des villes côtières se font les interprètes des puissances étrangères. Ainsi. Salomon Pariente au milieu du XVII' siècle est l'interprète de quatre 
gouverneurs britanniques qui se succèdent à Tanger pour y représenter la Couronne de Sa Majesté.

Ce statut privilégié d'interprètes va évoluer au cours des années, pour un petit nombre d'entre eux, vers celui d'agent puis de vice-consul et de consul. David Cohen cite un rapport du secrétaire de la légation de France à Tanger, datant de 1866, où il écrit à propos des juifs marocains:

Ils ont su conserver leur intelligence et leur activité. Courtiers habiles, ils sont devenus les intermédiaires presque indispensables entre les Européens et les Indigènes ${ }^{4}$.

Dans les années cinquante de ce siècle, il y avait encore à Rabat une rue commerçante de la ville indigène. à proximité du quartier juif, qui portait le nom de rue des Consuls, vestige de la période pré-coloniale où commerce et politique étrangère par le biais de la traduction n'étaient qu'une seule et même activité exercée au fond des boutiques et entrepôts par des importateurs de thé, de sucre et d'épices qui possédaient deux ou trois langues et certainement un sens aigu de la diplomatie.

Ces interprètes offraient leurs services à l'Angleterre, au Portugal, à l'Espagne, à l'Italie, à la Hollande. à la France et même aux États-Unis d'Amérique. Ils constituaient une classe privilégiée au sein de la population juive. Ils jouiront, eux et leurs descendants. parfois pendant des générations, d'un statut de protégés de ces puissances étrangères, ce qui leur permettra non seulement de faire fructifier leur négoce, mais aussi et surtout de se poser en défenseurs de leurs confrères juifs moins fortunés et moins influents lors des innombrables actes d'exaction anti-juifs perpétrés par des bandes armées incontrôlées ou incontrôlables par les chefs de tribus locales.

L'emploi d'étrangers dans la négociation des intérêts du pays est considérée. du moins en France, aux dires de Louis Féraud, comme funeste et justifie sous le Directoire, par le décret du 2 avril 1797, la création de l'École spéciale des langues orientales vivantes:

Outre l'enseignement les langues orientales, les professeurs feraient connaître à leurs élèves les rapports politiques et commerciaux de la France avec les nations dont ils enseignaient les langues.5. Maroc ${ }^{6}$

Le problème de l'emploi de ces étrangers indispensables se reposera également au

\section{La conquête}

La conquête de l'Algérie en 1830. soigneusement préparée, voit la constitution d'un corps d'interprètes militaires. Ce corps, composé d'orientalistes, d'élèves de l'École des langues orientales et d'officiers de mamelouks de l'ex-garde impériale comptait 40 interprètes qui se divisaient ainsi :

5 interprètes de $1^{\text {re }}$ classe:

3 interprètes de $2^{\mathrm{e}}$ classe :

7 interprètes de $3^{\mathrm{e}}$ classe :

25 guides-interprètes.

Ils étaient assimilés aux grades de colonel d'état-major, de chef d'escadron, de capitaine, et enfin de lieutenant, et avaient un uniforme de couleur bleu ciel dont la marque distinctive était une broderie en or de dcux branches d'olivier entrelacées. Par lettre du ministère de la Guerre en date du 5 avril 1830, les conditions matérielles étaient arrêtées, y compris les rations de fourrage pour leurs chevaux. au cas où le général en chef estimait nécessaire de leur en fournir. 
Le 25 mai 1830, quand la flotte quitte Toulon, ce n'est pas 40 interprètes qui l'accompagnent mais plus du double. En effet, l'effectif de 40 s'avérant insuffisant à la veille de l'expédition, l'état-major engage à Marseille et à Toulon des indigènes «accourus à la nouvelle du prochain départ». C'est ainsi que le nombre d'interprètes fut porté à 95 .

Parmi ce groupe démesurement grossi des guides-interprètes, et qui sera cause de bien des tracas, il faut signaler Abraham Daninos, juif originaire d'Alger, naturalisé français, vraisemblablement à la suite de services rendus à la France tels ceux décrits par L. C. Féraud. Nous le citerons largement ${ }^{7}$ :

Il avait été. pendant près de quatorze ans. interprète traducteur assermenté auprès du tribunal de commerce de la Seine, et, de plus, membre de la Societté asiatique de Paris.

Limprimerie royale et la poste genérale eurent de lui diverses traductions; il avait écrit divers ouvrages en langue française et arabe, et notamment un petit vocabulaire en langue vulgaire, qui fut distribué aux officiers qui firent partie de l'expédition d'Alger en 1830 .

Mandé par le ministre de la Guerre, il donna sur la ville d'Alger tous les renseignements qu'on lui demanda. et qu'il lui fut possible de donner à ceux qui assistèrent à la descente de nos troupes sur le sol africain.

Nommé guide-interprète en 1830, Abraham Daninos fut le plus puissant auxiliaire de $M$. Torpin, alors commandant de la frégate pilote ; et ce fut grâce à ses connaissances approfondies et à son infatigable zèle, que $M$. Torpin put operer des mouvements heureux. mouiller sans hésitation devant certains points, éviter des eaux dont il ignorait complétement la perfidie des courants.

Au cours de la conquête, les interprètes exerçaient souvent au péril de leur vie. Voici ce que Louis de Bracevitch, interprète devant le dey d'Alger, vécut le 4 juillet 1830 :

À ces mots, [article 3 de l'acte de capitulation «le Dey et les Turcs devront quitter Alger dans le plus bref délai»| un cri de rage retentit de toutes parts. Le Dey pâlit, se leva, et jeta autour de lui des regards inquiets. On n'entendait que les mots, répétés avec fureur par tous les janissaires : El mout ! el mout! (la mort! la morn!). Je me retoumai au bruit des yatagans et des poignards qu'on tirait des fourteaux, et je vis leurs lames briller au-dessus de ma tête. Je $m$ 'efforçai de conserver une contenance ferme. et je regardai fixement le Dey. Il comprit l'expression de mon regard, et prévoyant les malheurs qui allaient arriver, il descendit de son divan, s'avança d'un air furieux vers cette multitude effrénée, ordonna le silence d'une voix forte, et me fit signe de continuer. Ce ne fut pas sans peine que je fis entendre la suite de l'article, qui ramena un peu de calme $[\ldots \mid$ Comme je $n$ avais pas mission de traiter, mais de traduire et d'expliquer, je demandai à retoumer vers le général en chef, pour lui rendre compic de l'adhésion du Deyk.

Si Louis de Bracevich, soucieux de ne pas outrepasser ses fonctions d'interprète qui étaient de «traduire et expliquer» ne s'aventure pas à traiter, surtout dans de telles conditions, d'autres interprètes auront ordre de traiter, essentiellement à l'intérieur du pays. C'est ainsi que l'interprète Rémusat est chargé en 1835 de se rendre à Bône, Bougie et Mostaganem pour traiter la pacification du pays auprès du bey Ibrahim.

Nombre d'interprètes participent aux expéditions, comme René Baranes, Joseph Hamaouy: acquièrent une réputation de bravoure. comme Faradj Nakache et Léon Ayas, mort de suites de blessures; sont cités à l'ordre de l'armée d'Afrique, comme Moïse Adrey. Il convient de mentionner particulièrement Isaac Levy, natif de Gibraltar, fait prisonnier et mis à mort par Abd-el-Kader en 1845.

D'autres encore cuvrent dans l'ombre à collecter des renseignements sur l'ennemi, c'est ainsi que :

Adrien Hénon, né à Paris, sergent-interprète, feignant la désertion, il mena pendant plus d'un an une vie vagabonde de jongleur en vue de recueillir des renseignements sur les tribus alliées à Abd-el-Kader". 


\section{La mise en place des structures}

Une fois passée la période de la conquête, l'administration coloniale française en Afrique du Nord a opté pour une stratégie linguistique en trois temps, la première étant la reconnaissance des langues communes, à savoir le français, l'arabe et les autres langues pratiquées par les indigènes (berbère, judéo-arabe, hébreu, maltais, etc.).

Cette reconnaissance avait pour corollaire le recours aux services de traducteursinterprètes. Voici ce qu'en dit un magistrat algérien dans une postface anonyme à l'ouvrage de Féraud, intitulée De l'interprétation et rédigée sous forme de loi comprenant un préambule et 61 articles:

Sur les trente mille Hébreux de l'Algérie. combien qui savent lire. écrire et parler le français? Et parmi les interprètes pour la langue arabe, combien qui savent lire, ecrire et parler l'hébreu? Donc, partout où une agglomération isrálite la commande, création, sous les mêmes conditions, mutatis mutandis, que les autres interprètes, d'interprètes pour la langue hébraïque! De même, et à plus forte raison, pour la langue kabyle ${ }^{10}$.

Puis, dans un deuxième temps, est intervenue la distinction entre les langues passives, ou langues de la réception comme l'arabe, et la langue active, celle de l'émission, le français.

La lettre du duc de Rovigo au maréchal Bugeaud en date du 1.5 octobre 18.32 expose déjà le programme de colonisation linguistique dans ses grandes lignes. Nous en citerons de larges extraits:

\footnotetext{
...La Régence d’Alger ne sera véritablement une possession française, que lorsque notre langue sera nationalisée, et que les arts et les sciences, qui font la gloire de notre patrie. y seront acclimates. |... Trois institutions françaises sont déjà établies a Alger. et le nombre total des enfants qui les fréquentent est de quatre-vingt-dix

Ce nombre ira chaque jour en augmentant. Une chaire de langue française vient d'être créée pour les juifs indigènes : 4) élèves y reçoivent des leçons, trois fois par semaine. J'ai l'espoir de voir bientôt leur nombre doublé : leurs progrès sont remarquables.

Un local sera mis, sous peu de jours, à la disposition de l'inspecteur de l'instruction publique, pour l'établissement d'une classe de jeunes maures qui seront initiés a la connaissance de notre langue. Dans ce même local, une chaire sera fondée pour l'enseignement de la langue arabe aux Européens, sous la direction intelligente de l'interprète militaire Joanny Pharaon. afin de rendre plus faciles et plus promptes les communications entre nous et les indigènes. Le vrai prodige à operer serait de remplacer peu à peu l'arahe par le français. qui. étant la langue des autorités et de l'administration. ne peut manquer de s'étendre parmi les indigènes, surtout si la génération nouvelle vient en foule s"instruire dans nos écoles 11 .
}

Enfin, la dernière phase linguistique est celle de l'imposition de la langue du dominant sur la/les langues des dominés. Ce troisième stade qui voit la réduction de toutes les langues en présence à une seule langue, «formule la plus rationnelle et la plus économique, à défaut d'être la plus simple en termes politiques". comme l'affirme J. Lambert'2 - et que L. J. Calvet qualific de stade glottophage où l'européen a dévoré les langues du colonisé : «D'ailleurs, les langues n'existaient pas, n'est-ce-pas. Tout juste des dialectes, voire des patois. s1.3 - se fera avec l'aide des interprètes militaires, comme nous venons de le voir.

L'activité traduisante n'interviendra dans celte phase que dans le cadre éducatif de l'apprentissage de la langue dans le but de s'assurer une bonne compréhension de la langue à acquérir. en tout premier lieu dans le domaine militaire, tel que Gisèle Kahn le montre en notant la présence indispensable de l'interprète aux côtés de l'instructeurenseignant :

Au début, le gradé européen n'est pas compris; il fait appel, pour traduire ses ordres, à un gradé indigène (ou à un tirailleur ancien) parlant l'idiome du tirailleur interpellé 14. 
La mise en place de structures administratives, judiciaires et autres devant prendre le relais des structures militaires de la conquête, les besoins en traduction d'actes seront remplis par d'anciens interprètes militaires. C'est parmi ces derniers que l'on verra apparaître, à partir de 1870 . les premiers écrivains algériens musulmans de langue française's.

Pour la Tunisie et le Maroc, la période d'activité des traducteurs est en même temps plus diffuse et plus longue dans sa période pré-coloniale, mais plus courte dans sa phase militaire, de par la nature de la conquête de ces pays. La proximité géographique de ces deux pays situés de part et d'autre de l'Algérie. l'antériorité de la conquête d'Alger et la diffusion du français par des organismes tels que l'Alliance israélite universelle ont fait que l'intervention des traducteurs a été moins spectaculaire dans les pays limitrophes de l’Algérie.

\section{PORTRAIT DES TRADUCTEURS}

Nous l'avons déjà remarqué, les traducteurs-interprètes ne forment pas un bloc monolithique, loin de là. De par leur origine et leur formation, ils constituent deux groupes distincts qui ont entretenu entre eux des relations qui ont pu être des relations d'ignorance réciproque, des relations de coopération ou des relations litigieuses. Leurs champs d'action ont été différents de même que leurs relations au pouvoir.

\section{Des origines bien distinctes}

Si au moment de l'embarquement à Toulon le corps des quarante interprètes engagés des mois auparavant était renforcé ou plutôt affaibli par une cinquantaine de guidesinterprètes recrutés à la demière minute, cette hétérogénéité ira en s'accentuant sur le terrain.

Les interprètes militaires de l'armée d'Alger étaient tous des «individualités d'élite occupant déjà, pour la plupart, des positions lucratives, que l'on allait déranger de leurs travaux ou de leurs habitudes, pour entrer en campagne" et à qui l'on se devait "certains Aménagements» 16 .

Les guides-interprètes sont au contraire des indigènes autodidactes qui avaient acquis une connaissance du français par l'exercice du commerce ou d'activités semblables. La majorité d'entre eux sont juifs et entrent au service de la France alors qu'ils ont entre quinze et vingt ans. Ils graviront lentement les échelons et peu d'entre eux accèderont au statut enviable de traducteur titulaire auquel ne parvinrent que ceux qui, particulièrement brillants, purent obtenir la naturalisation française.

Bien que leurs conditions d'avancement aient été réglementées par un examen annuel comportant des épreuves de langue, d'histoire, de géographie et d'arithmétique, ils demeuraient «ces sortes d'interprètes». «des agents secondaires pour faciliter le service». "des sujets dont l'instruction n'est pas toujours à la hauteur de leur position», "des hommes sans intelligence et sans dignité».

Un des signes extérieurs distinctifs entre ces deux groupes que tout séparait était le port de l'uniforme. Or, certains abus se produisirent. Notamment lorsqu'un de ces guidesinterprètes fut arrêté en état d'ébriété à la suite d'une querelle au sortir d'un tripot du port d'Alger alors qu'il revêtait l'uniforme.

Ce sont des événements semblables qui, estime Louis Féraud, ont provoqué le départ de beaucoup d'interprètes dès la fin de l'année 1830:

...une raison bien autrement grave les déterminait à renoncer à leurs fonctions : c'était le profond dégoût qu'ils éprouvaient, eux hommes d'honneur, de se voir confondus, dans l'opinion publique, avec certains guides-interprètes, ramassés trop à la hâte sur les quais de Toulon, qui avaient déjà abusé de leur position pour se livrer à des actes ignominieux ${ }^{17}$.

Le retour en France de bon nombre d'interprètes de carrière, les besoins grandissants de l'armée et de l'administration auront pour conséquence l'augmentation du nombre de 
guides-interprètes. De la composition du corps entre les années 1830 et 1870 el à travers les nombreuses réorganisations et même régénérations qu il a connues, il ressont que les interprètes de carrière constitueront de plus en plus les cadres de ce corps, alors que le gros de l'effectif sera composé de guides indigènes.

Ces derniers, juifs dans leur grande majorité au début ( 24 juifs et 8 musulmans), céderont leur place aux musulmans qui se feront plus nombreux $(5$ juifs et 9 musulmans en 1876) sans pour autant devenir l'élément prédominant puisqu'on note dans leurs rangs, toujours en 1876, 23 guides-interprètes français nés en Algérie.

\section{Les qualités professionnelles requises}

Outre les qualités civiques et intellectuelles, elles sont de deux ordres qui aujourd'hui ne paraissent pas devoir être nécessairement réunies chez un même interprète, à savoir les qualités guerrières et le sens de la diplomatic. Voici l'éloge que fait le maréchal Bugeaud de l'interprète en chef de l'armée Léon Roches, dans une lettre au ministre des Affaires étrangères en date du 15 juillet 1845 :

Il exerce un tel prestige sur les Arabes, que j’ai vu maintes fois, des populations entières, ramenées par lui seul à la soumission. - C'est qu'il parle et écrit l'arabe, comme un lettré musulman; qu'il a l'aspece guerrier: qu'il égale l'habileté des meilleurs cavaliers arabes, et que son courage est devenu proverbial parmi eux. Jajoute que son esprit ardent. son cour généreux et prompt au dévouement, le rendent séduisant; c’est en outre un excellent Français 18

Les délicates et importantes fonctions de confiance à remplir pour lesquelles les interprètes de l'armée d'Afrique étaient engagés comprenaient, selon Louis Féraud, aussi bien le service de la correspondance arabe que les liens à nouer avec les indigènes ou, au besoin, la reconnaissance en pays inconnus. Aussi, on exigeait d'eux probité el instruction. Dans un rapport adressé en 1854 par le maréchal A. de Saint-Arnaud, ministre Secrétaire d'État de la Guerre à l'Empereur, on peut lire:

Les fonctions de l'interprétation, en rendant un agent l'intermédiaire obligé de mesures politiques ou de plans d'opérations militaires, exigent naturellement qu elles ne soient accordées qu'à des nationaux et à des hommes sur la fidélité et la discrétion desquels on puisse compter.

Quant à Napoléon, toujours d’après Louis Féraud qui était lui-même interprète principal du corps en 1876 :

Il pensait que par la nature de ses fonctions. l'interprète est un homme fidèle el consciencieux, l'intermédiaire obligé de toute négexiation: que la parole qui passe par sa bouche doit arriver pure de toule arrière-pensée. L'interprète est l'homme de confiance : if double le personnage qu'il sert. Il doit étudier son caractère, ses habitudes, car il ne s'agit pas en traduisant, de rendre des paroles seulement, mais encore toutes les nuances de la pensée ${ }^{19}$.

Féraud, dans un grand élan lyrique, dira des interprètes qu ils sont de véritables missionnaires, qu'ils pratiquent la vraie charité chrétienne et n’hésitera pas à les comparer à «nos pères dans le Canada».

\section{Des voies divergentes}

Les interprètes de l'armée d'Afrique connaîtront des voies divergentes. Leur rêinsertion après le service dans les colonies se présente différemment selon leur origine et selon leur inclination.

Parmi ceux qui rentrent en France et reprennent leur carrière scientifique, il faut signaler Charles Brosselard qui publie en 1844. à l'Imprimerie royale, un dictionnaire berbère. Mais «l'illustration scientifique dont $s$ 'honore le plus le corps des interprètes de 
l'armée» est le baron William Mac-Guckin de Slane, d'origine irlandaise, auteur de la traduction commentée des Prolégomènes d'Ibn Khaldun sur I'histoire des Berbères 20.

Il sera également titulaire de la chaire d'arabe à l'École spéciale des langues orientales de Paris. D'autres, comme lui, choisissent la voie de l'enseignement à Marseille, à Alger.

La carrière diplomatique se présente souvent comme une issue prestigieuse à ceux qui ont fait la preuve éclatante de leurs qualités. Nombre d'anciens interprètes seront nommés consuls en Afrique du Nord et dans ce qu'on appelait alors le Levant. Comme l'écrivait le maréchal Bugeaud à M. Guizot, ministre des Affaires étrangères dans sa lettre du 15 juillet 1845, précédemment citée : «Il faut savoir prendre les hommes, propres à la chose, là où ils sont, et faire fléchir les règles hiérarchiques devant un grand intérêt national. Pour ceux qui resteront en Algérie, le corps deviendra de façon naturelle "la pépinière' où se développeront un bon nombre d'agents propres aux divers services judiciaires et civils $21 . »$

C'est en effet parmi eux que seront recrutés par exemple le Lieutenant général de la police ainsi que le Commissaire général et le Directeur des domaines.

Enfin, la carrière militaire fera d'anciens interprètes des officiers avec des grades de lieutenant-colonel, chef d'escadron, capitaine de cavalerie, etc.

Quant aux interprètes indigènes, certains d'entre eux parvinrent à des fonctions d'interprète judiciaire, comme Abraham Daninos, ou à celles de consul, comme Emmanuel Nahon, qui avait été interprète de l'armée et de l'administration civile pendant vingt et un ans, avait participé à 14 campagnes et avait été blessé à plusieurs reprises. Mais ce type de promotion, rarissime s'il en fût, sera l'objet d'une étude ultérieure 22.

Pour les uns comme pour les autres, mais vraisemblablement plus pour les interprètes français, le passage en Algérie n'aura pas été inutile sur le plan professionnel:

Il y a une très grande différence entre l'orientaliste qui travaille à loisir dans un commode cabinet, abrité du chaud et du froid, et l'interprète militaire qui fait de la traduction orale ou écrite, sans livres, à cheval ou sous la tente, ou même sans tente, et exposé à toutes les rigueurs des saisons. Il est vrai qu'un grand avantage compense cet inconvénient: car si l'interprète militaire, placé dans les circonstances que nous venons d'indiquer, peut difficilement perfectionner et accroître ses connaissances théoriques, il est à même de faire d'importantes études pratiques, qui seraient impossibles ailleurs. La conséquence naturelle de ce qui précède paraît être qu'il faut soumettre les interprètes de l'armée à un roulement qui les fasse passer successivement d'une position à l'autre, afin que chacun ait, à son tour, l'occasion de cultiver, sous leurs deux faces - pratique et théorique - les connaissances spéciales requises dans leur difficile profession 2.3 .

\section{UNE DIFFICILE PROFESSION}

Les interprètes de l'armée d'Afrique sont dénommés, nous l'avons vu, aussi bien par l'Empereur, par le ministre de la Guerre que par le Résident général de la République française à Tunis par l'expression intermédiaire obligé. Ceci n'est certainement pas fortuit ${ }^{24}$.

Il convient de se demander ce que révèle cette expression. Sur quelle conception de la colonisation s'appuie-t-elle?

A-1-elle été forgée par une conception mercantile, institutionnelle ou idéologique de la colonisation et du rôle du traducteur et de la langue dans le processus colonisateur?

Elle ne peut en tout cas relever d'une conception neutre de la langue et partant de la traduction comme communication. Ici, la langue est instrument de puissance. Le traducteur, peut-il alors se contenter d'être cet énonciateur des rapports de forces en présence? La pratique de son art dans ce contexte ne risque-t-elle pas de le pervertir? Son activité ne risque-t-elle pas de friser la subversion en instituant entre ses langues de travail des rapports de dominant à dominé ? 
C'est peut-être plus ce type de danger que la crainte de voir leur statut déprécié par la présence de guides-interprètes qui a poussé nombre d'interprètes à regagner la France dès la fin de l'année 1830, provoquant ainsi une crise que le corps aura beaucoup de mal à surmonter et dont le souvenir était encore vivace quarante ans plus tard.

Ou alors, cette interprétation n'est-elle que le fruit d'une pensée qui projette une conception décolonisée de la traduction?

Cependant, un fait reste certain. Puisque notre devoir de chercheur est comme le dit J. Lambert de "déceler la nature et l'enjeu des conflits 25 ", il faut bien dire qu'être traducteur dans un contexte colonisateur, $c$ 'est être au centre d'un conflit de valeurs, c'est personnaliser ce conflit par langues interposées et parfois même payer de sa vie. comme ce fut le cas pour Salomon Pariente à Tanger au XVIIc siècle et pour Moses Azoulay à Tétouan à la fin du XIXe siècle.

\section{Notes}

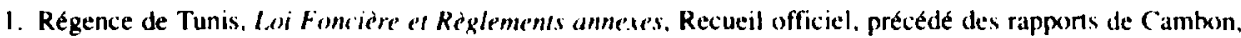
P. et Massicault, M., Paris. Challamel, 1893.

2. LAREDO. A. I. (1978) : le's noms des juifs du Maror, Consejo Superior de Investigaciones Cientificas, Madrid, Instituto. B. Arias Montano.

3. HIRSHBERG (1981): A Histery of the It'u's in North Afruca. Lid. II. From the Ottoman Conquests to the Present Time, second revised edition, translated from the Hebrew, edited by Eliezer Bashan and Roben Attal, Leiden, E. J. Brill.

4. COHEN, D. (1980): “Les communautés juives des villes côtières du Marox entre 1880 et 1940". Juifs dh Marse - Identité et Dialogue. Paris. Éditions de lat Pensece sauvage.

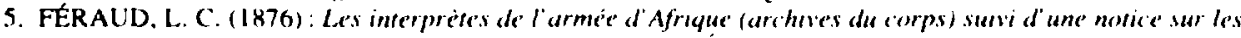
interprestes civils et judic tuires.s. Alger, A. Jourdan. Libraire-Éditeur.

6. Il est intéressant de signaler à ce propos la série d'articles de mars 1895 dans le périodique la révell de Maror sous le titre: «le service consulatre au Martén dont la publication sera intertompue. Vraisemblablement. l'auteur anonyme ne réussit pas à alteindre l'objectif yu'il s'ćtait fixé

En entreprenant celte étude. beaucoup plus épineuse qu'on ne pense, mous faisons un varu. c'est celui de pouvoir faire ressortir tous les inconvénents de la situatıon que nous dénonçons. sans frossser directement personne et satms etre ohlige de rappeler eci certaims faits qui, tout en vervant de corollaire a nos appréciations. $n^{\prime}$ auraient rien de flatteur peur cette civilisation que nous prétendons impeser aux Marocains ni pour ses représentants les plus accredites dans ce pays,

La critique ouverte sur les bientaits civilisateurs de la colonisattion avant de quoi déplaire aux lecteurs de cet hebdomadaire qui appelaient de tous leurs vorux l'arrivée des Français au Marrex.

7. FÉRAUD. (op. (it. pp. 19()-191.

8. Cité par M. Merle, secrétaire paniculier du général de Bourmont. dans: Anerdones histornques sur les conquête's d'Alger e'n /8.30. d'apres Féraud.

9. FERAIID, op. ilf.pp. 294 it 297.

10. FERAUD, op. (it. p. $46(x)$, article 57

11. FERAUD, op cit. pp. 230-231. (ité également mais succonctement par (alvet et Achour (voir plus bas, notes 1.3 et 15$)$

12. LAMBERT. J. (1989): “l.a traduction. les langues et la communication de masse. Les ambiguîtés du discours internationalm. Targe't, 1-2. pp. 215 a 237.

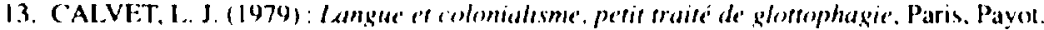

14. KAHN, (i. (1990) : “l'n manuel pour l'enseignement du français aux militaires indigènes. 1927». I. françats dans le monde' numéro spécial : Publics spécifiques et communication spécialiséc. Paris. Hachette. pp. 97 a 103.

15. ACHOUR. C. (1990) : «Pour une hısioire du françain en colonie - le cas de l'Algérie», Études de lingıeis. tique appliquée, $\mathrm{n}^{\circ} 78$, Paris, Didier, pp. 87 à 94.

16. FÉRAUD, op. (1t., p. 48

17. FERAUD, op. (II. p. 62

18. FÉRAIJD. op (it. pp. 242-243

19. FÉRAUD, op cit, p. 81

20. FERAUD, op. ca., p. 288 et A. CAQUOT (1989) «Deux siecles d'orientalisme", Déu siecles de France. Total Infornation $\mathrm{n}^{\mathrm{t}} 111$.

21. Extrait du rapporn de M. Biesnier. membre du jury d'examen des interprètes en 1859, cité par Féraud p. 137.

22. (ff son dossier aux Archives diplomatiquer du qual d'Orsay. 
23. Extrait de l'allocution du président de la Société historique algérienne fondé en 1856, rapponté par Féraud.

24. On retrouve la méme expression dans le rapport de Freycinet au President de la Republique le 18 décembre 1880: «Les drogmans sont en Orient, les intermediaires obliges de nos agents diplomatiques et consulaires auprès des autorites tertitoriales: les interprètes jouent le même rôle en Extrème-Orient". Les affaires etrangères el le corps diplomatique français. (1984) CNRS. tome 2. p. 162.

25. Op. cit. note 12, p. 225. 